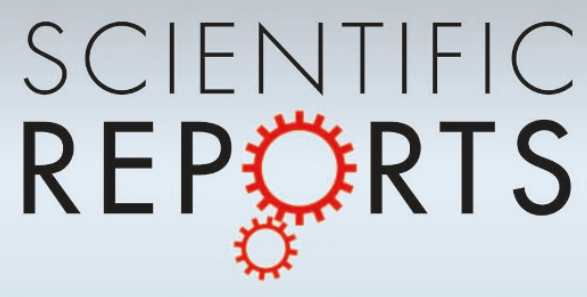

\title{
OPEN \\ SUBJECT AREAS: \\ SOLAR CELLS \\ Interface engineering of graphene for universal applications as both anode and cathode in organic photovoltaics
}

GRAPHENE

NANOSCIENCE AND

TECHNOLOGY

OPTICAL PROPERTIES AND

DEVICES

\author{
Hyesung Park',2, Sehoon Chang ${ }^{2}$, Matthew Smith², Silvija Gradečak² \& Jing Kong'
}

'Department of Electrical Engineering and Computer Science, Massachusetts Institute of Technology, Cambridge, Massachusetts 02139, USA, ${ }^{2}$ Department of Materials Science and Engineering, Massachusetts Institute of Technology, Cambridge, Massachusetts, 02139 USA.

Received

27 December 2012

Accepted

13 March 2013

Published

2 April 2013

Correspondence and requests for materials should be addressed to

J.K. (jingkong@mit.

edu)
The high transparency of graphene, together with its good electrical conductivity and mechanical robustness, enable its use as transparent electrodes in optoelectronic devices such as solar cells. While initial demonstrations of graphene-based organic photovoltaics (OPV) have been promising, realization of scalable technologies remains challenging due to their performance and, critically, poor device reproducibility and yield. In this work, we demonstrate by engineering the interface between graphene and organic layers, device performance and yield become close to devices using indium tin oxide. Our study confirms that the key issue leading to the poor performance or irreproducibility in graphene-based OPV originates from the graphene interface, and can be addressed by a simple interface modification method introduced in this work. We also show similar approach allows graphene to be used as cathode in inverted OPV geometry, thereby demonstrating the universal application of graphene as transparent conductors for both the anode and cathode.

raphene, a hexagonal arrangement of carbon atoms forming a one-atom thick planar sheet, possesses unique and outstanding electrical ${ }^{1-3}$, mechanical ${ }^{4}$, optical ${ }^{5}$, and chemical $^{6}$ properties. As a building block for carbon materials of all other dimensionalities, two-dimensional graphene has been widely studied by theorists since the middle of the last century ${ }^{7,8}$. Yet it was not until the successful isolation of single- and few-layer graphene by the mechanical cleaving of highly ordered pyrolytic graphite ${ }^{9}$ that graphene began to attract widespread attention. More recently, the continuous and scalable large area synthesis of graphene by chemical vapor deposition $(\mathrm{CVD})^{10,11}$ has bolstered its study in a broad range of research areas ${ }^{12-15}$. Notably, there has been a growing interest in the potential of graphene-based optoelectronic applications ${ }^{16}$ taking advantage of its high transparency, good electrical conductivity, and mechanical robustness. In particular, initial demonstrations of graphene as a replacement transparent electrode for indium tin oxide (ITO) in organic photovoltaic (OPV) devices $^{13,17-19}$ have been promising. However, in these studies the performance of graphene devices generally remains lower than that of their ITO counterparts and, more importantly, device yields have been low ${ }^{13,17-19}$; a crucial issue that must be solved if 'real-world' applications are to come to fruition.

In OPV solar cells, ITO electrodes are usually interfaced by a thin layer of conducting polymer, poly $(3,4-$ ethylenedioxythiophene):poly(styrenesulfonate) (PEDOT:PSS). The PEDOT:PSS (Clevios ${ }^{\text {TM }}$ P VP AI 4083$)^{20}$ hole injection layer (HIL), with a work function of $5.2 \mathrm{eV}$, is understood to improve overall device performance by: (1) facilitating the injection/extraction of holes via an ohmic-contact at the junction; (2) planarizing ITO surface roughness, which otherwise presents potential sources of local shunting through the device's ultra-thin active layers ${ }^{21,22}$; and (3) inhibiting dewetting of the overlying organic layers by providing a more chemically compatible electrode surface than ITO. In order to achieve uniform coverage of PEDOT:PSS on ITO, ITO electrodes are typically treated with an oxygen plasma prior to spin-coating of PEDOT:PSS so as to increase their hydrophilicity. However, achieving smooth and complete coverage of PEDOT:PSS from aqueous solution onto graphene is challenging owing to the surface's hydrophobic nature ${ }^{23}$. Previous efforts ${ }^{13,24}$ have mostly employed destructive treatments, such as with $\mathrm{O}_{2}$ plasma or UV-ozone, on thicker multilayer graphene electrodes, to convert the graphene surface from hydrophobic to hydrophilic ${ }^{25}$, but these processes greatly reduce the electrical conductivity of graphene. Previously ${ }^{17}$, we have found that without a proper HIL, graphene-based OPV solar cells do not exhibit diode-like rectification, but instead show resistor-like behavior with very limited photoresponse. Thus it has been a continuous effort to either improve the coverage uniformity of PEDOT:PSS layer on 
graphene electrodes or replace PEDOT:PSS by other layers of similar function. Toward this end, doping by $\mathrm{AuCl}_{3}{ }^{13}$, replacing PEDOT:PSS by inorganic $\mathrm{MoO}_{3}{ }^{17}$ or vapor deposited PEDOT layer ${ }^{26}$, or using solution modified PEDOT:PSS ${ }^{27}$ has been investigated and the device yield has been continuously improving. Furthermore, although not studied with graphene electrodes, several works reported modifying the PEDOT:PSS solution via various surfactants to improve the wetting property on a variety of hydrophobic substrates ${ }^{28-30}$. Nevertheless, the device yield obtained from the previous methods have been $30-40 \%{ }^{13,17,26}$. In this work, by engineering the interfacial HIL between the graphene electrode and the organic donor layer, we find that the performance and yield of graphene based-OPV devices become close to those of devices using ITO electrodes. This interface modification is achieved by inserting a thin polymeric buffer layer between the pristine graphene surface and the PEDOT:PSS HIL, thus forming a Double-HIL structure (D-HIL). We also demonstrate that this method can be further expanded to the inverted solar cell geometry where graphene is utilized as the cathode, thus illustrating the broad application of graphene as a transparent conductor in OPV in general.

\section{Results}

To form a D-HIL structure, we use poly(3,4-ethylenedioxythiophene)-block-poly(ethylene glycol) (PEDOT:PEG) doped with perchlorate (PC) in nitromethane $\left(\mathrm{CH}_{3} \mathrm{NO}_{2}\right)(1 \mathrm{wt} . \%)$ as the buffer layer (which we refer to as PEDOT:PEG(PC)), which is spin-coated onto graphene prior to PEDOT:PSS deposition. The molecular structures of PEDOT:PEG(PC) and PEDOT:PSS are compared in Fig. 1a, and Fig. $1 \mathrm{~b}$ shows the transmittance of the spin-coated films on quartz substrates. Although PEDOT:PEG(PC) is less transparent than PEDOT:PSS $(88.5 \% v$ s. $97.5 \%$, at $\lambda=550 \mathrm{~nm}$ incident light wavelength), the difference in transparency does not limit the device performance, as shown later. The average sheet resistances of PEDOT: PEG(PC) and PEDOT:PSS on quartz were $25 \pm 4 \mathrm{M} \Omega /$ sq and $17 \pm$ $3 \mathrm{M} \Omega / \mathrm{sq}$ at thicknesses of $40 \mathrm{~nm}$ and $20 \mathrm{~nm}$, respectively. Replacing PSS with PEG, which co-polymerizes the PEDOT with PEG, renders PEDOT soluble in organic solvents and enables it to efficiently wet the hydrophobic surface of graphene, as well as other substrates onto which spin-coating of uniform PEDOT:PSS is difficult.

This surface modification is confirmed by characterization of surface morphologies. The spin-coated PEDOT:PEG(PC) completely covers the graphene surface, in contrast to PEDOT:PSS, which coats graphene non-uniformly as shown in the optical micrograph from Fig. 2a, b. The better-wettability of PEDOT:PEG(PC) on graphene can be also observed from the contact angle measurement. Microscale droplets of both solutions, PEDOT:PEG(PC) and PEDOT:PSS, were placed on graphene transferred on quartz substrates to investigate the wettability and the static contact angle. As shown in Fig. 2c,d, the contact angles for PEDOT:PEG(PC) and PEDOT:PSS are $28.1 \pm 1.4^{\circ}$ and $91.2 \pm 0.2^{\circ}$, respectively. Further characterizations of the D-HIL films are provided in the Supplementary Fig. S1 from the scanning electron microscopy (SEM) and atomic force microscopy (AFM). SEM images show that, although the PEDOT: PEG(PC) surface is rougher than that of PEDOT:PSS, it is smoothened by the subsequent coating with PEDOT:PSS (Supplementary Fig. S1b, d). And the observed roughness did not appear to affect device performance as shown later.

In addition to facilitating the deposition of high quality PEDOT: PSS on graphene electrodes, it is noteworthy that the PEDOT: PEG(PC) buffer layer is much less corrosive to the underlying electrode than PEDOT:PSS: Jong et al. ${ }^{31}$ reported that PEDOT:PSS, with a $\mathrm{pH}$ value of 1-2 (due to the strongly acidic nature of PSS), etches indium out of ITO anodes, which can contribute to the diffusion of indium atoms into the active layers of an OPV or organic lightemitting diode (OLED), reducing their operating lifetime. Indeed, indium diffusion has commonly been linked to the degradation of OLEDs $^{32}$.

By incorporating the D-HIL structure, we demonstrate archetypical bi-layer heterojunction small molecule solar cells based on pristine (i.e. no doping or chemical treatment) graphene electrodes with performance and yields close to ITO reference devices. The device architecture employed was anode graphene (or ITO)/D-HIL (PEDOT: PEG(PC)-PEDOT:PSS)/tetraphenyldibenzoperiflanthene (DBP, $25 \mathrm{~nm}) /$ fullerene $\left(\mathrm{C}_{60}, 40 \mathrm{~nm}\right) /$ bathocuproine $(\mathrm{BCP}, 8.5 \mathrm{~nm}) / \mathrm{Al}$ $(100 \mathrm{~nm})$. Graphene films were synthesized under low pressure CVD conditions and the graphene electrodes were prepared via layer-by-layer transfer ${ }^{17}$ by stacking three monolayers of graphene sheets: the resulting average sheet resistance $\left(R_{s h}\right)$ and transmittance values were $300 \pm 12 \Omega / \mathrm{sq}$ and $91.8 \pm 0.4 \%$ (at $\lambda=550 \mathrm{~nm}$ ), respectively. Fabrication and testing procedures are detailed in the Methods sections. A schematic of the device structure, as well as a transmission electron microscopy (TEM) image of its cross-section, a

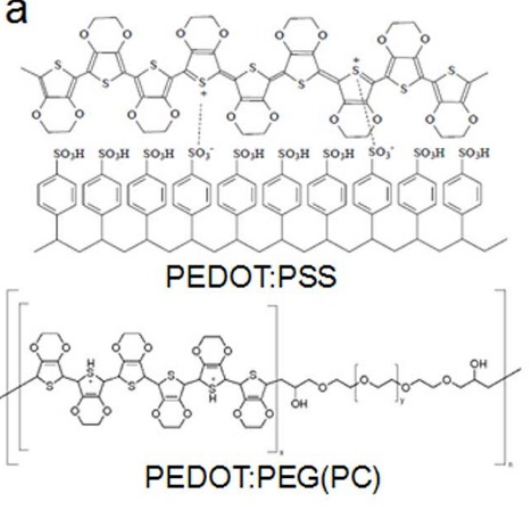

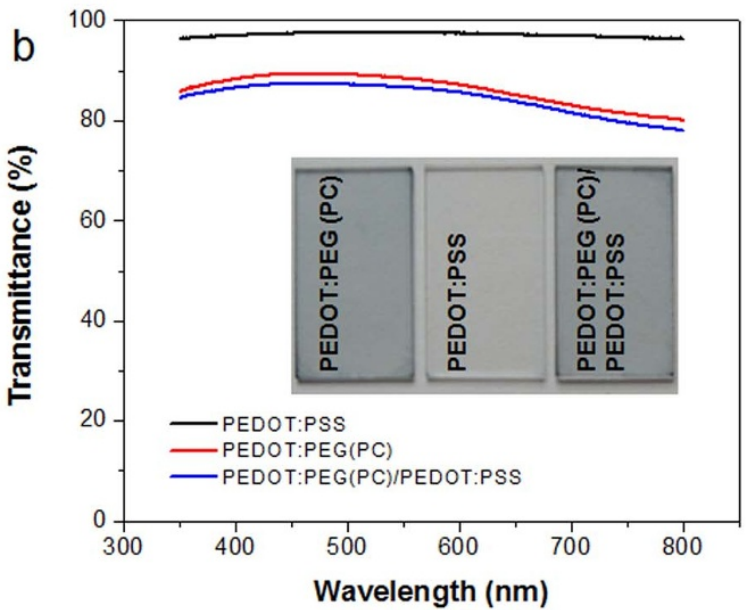

Figure $1 \mid$ Characterizations of the D-HIL structure. (a) Molecular structures of PEDOT:PEG(PC) and PEDOT:PSS. (b) Ultraviolet-visible spectroscopy transmittance spectra of each of the polymeric layers and of the double-layer, all spin-coated on quartz substrates. PEDOT:PEG(PC) and PEDOT:PSS layers were each spin-coated at $4000 \mathrm{rpm}$, resulting in film thicknesses of $40 \mathrm{~nm}$ and $20 \mathrm{~nm}$, respectively, as measured by atomic force microscopy. Varying the spin speed (1500-5000 rpm) of PEDOT:PEG(PC) did not significantly affect its transmittance ( $<5 \%$ variation), film thicknesses $(<10 \%$ variation) or sheet resistance ( $<5 \%$ variation). The D-HIL was formed by consecutive spin coating of PEDOT:PEG(PC) and PEDOT:PSS. The final D-HIL film thickness is $55 \mathrm{~nm}$. The inset shows macroscopic photographs of each polymer film spin-coated on glass substrate. 


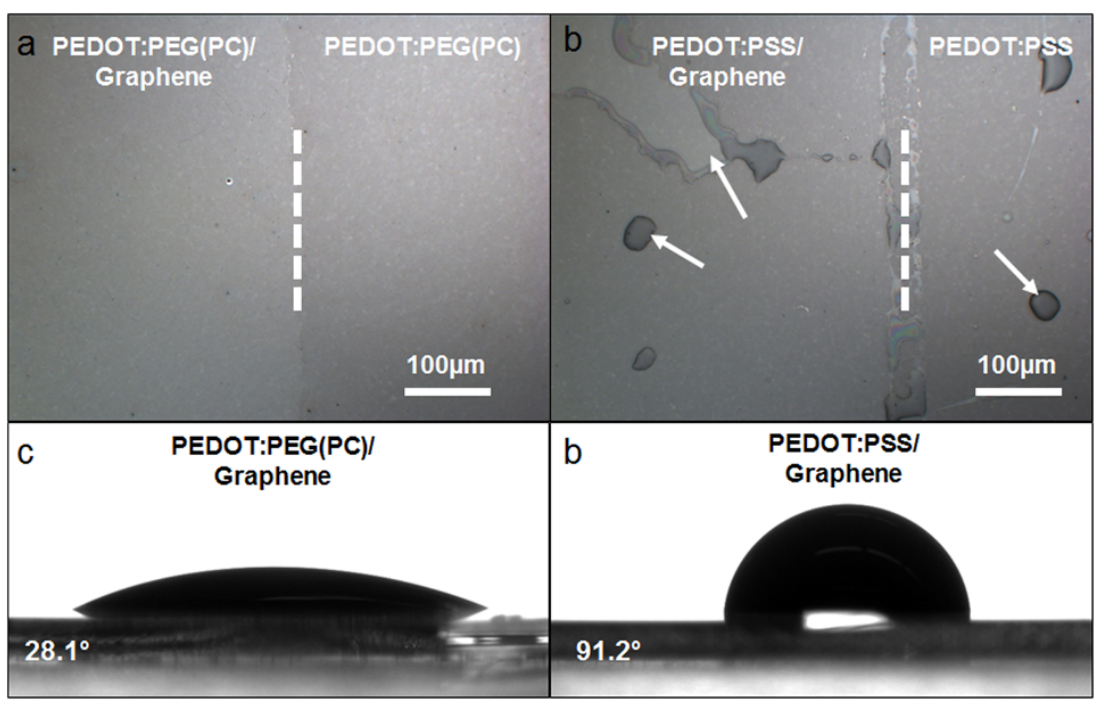

Figure $2 \mid$ Hole injection layer wetting properties on graphene surface. Bright-field optical microscopy images of (a) PEDOT:PEG(PC) on graphene and bare quartz and (b) PEDOT:PSS on graphene and on bare quartz substrates. The white dotted lines indicate the edge of the graphene and the arrows denote dewetted PEDOT:PSS. Contact angle images of (c) graphene/PEDOT:PEG(PC) and (d) graphene/PEDOT:PSS. Figures (a) and (c) illustrate better wettability of the PEDOT:PEG(PC) on graphene surface than PEDOT:PSS on graphene.

is shown in Fig. 3a, b, along with the corresponding flat-band energy levels of each material in Fig. 3c. The results of energy dispersive Xray spectroscopy (EDS) are shown on the right panel of Fig. 3b, identifying the materials corresponding to the cross-sectional TEM image and confirming the targeted structure.

Figure $3 \mathrm{~d}$ illustrates the current density-voltage $(J-V)$ results for the solar cells described above. ITO-based reference devices with the same structure (i.e. with a D-HIL) or with only a PEDOT:PSS HIL are shown for comparison. Devices using the D-HIL structure, with either ITO or graphene electrodes, display rectifying $J-V$ characteristics similar to those of the ITO/PEDOT:PSS control device. The key photovoltaic parameters of the representative devices (short-circuit current density $\left(\mathrm{J}_{\mathrm{SC}}\right)$, open-circuit voltage $\left(\mathrm{V}_{\mathrm{OC}}\right)$, and fill factor $(\mathrm{FF})$ ) are summarized in Supplementary Table S1. All three solar cells show similar power conversion efficiency (PCE): $2.9 \%$ for graphene/DHIL; 3.2\% for ITO/D-HIL; and 3.1\% for ITO/PEDOT:PSS. Similar cell performance of ITO reference device was reported previously ${ }^{33}$. It is evident that the type of HIL, i.e., single- or D-HIL, has negligible impact on ITO-based devices. This is somewhat surprising given that the D-HIL is actually 2 times thicker than the single-HIL.

PEDOT:PEG(PC) alone, however, is not suitable for mediating hole injection between graphene and an adjacent donor material due to its lower work function $(4.3 \mathrm{eV})$ than PEDOT:PSS $(5.2 \mathrm{eV})$. Moreover, the rough surface of PEDOT:PEG(PC) can be possible source of the shorting. Indeed, the resulting solar cell fabricated with a PEDOT:PEG(PC)-only shows non-rectifying device characteristics (Supplementary Fig. S2a). At the same time, solar cells with graphene electrodes with only spin-coated PEDOT:PSS HIL (i.e. devices exhibiting poor HIL wetting on graphene) show almost resistor-like behavior, similar to the behavior of devices without any HIL (but typically with slightly better power conversion efficiency (PCE)) (see Supplementary Fig. S2b). On the other hand, when PEDOT:PEG(PC) is used as an intermediate buffer layer between graphene electrode and PEDOT:PSS, the photo-response of devices using the D-HIL configuration is significantly improved. This D-HIL serves two roles: the PEDOT:PEG(PC) layer facilitates the wetting of the PEDOT:PSS HIL on graphene; while the PEDOT:PSS layer, with its higher work function and high carrier density, serves as an energetic intermediate between graphene's shallower Fermi level and the donor material's deeper highest occupied molecular orbital (HOMO). This D-HIL structure, prepared by simple spin-coating in ambient condition, allows PEDOT to be deposited onto graphene electrodes without the introduction of defect sites (in contrast to the $\mathrm{O}_{2}$ plasma or UVozone treatment $\left.{ }^{13,24}\right)$. Additionally, this approach does not require any unstable modifications (e.g., chemical doping) of the graphene surface, and could therefore be applied even to single layer graphene. The D-HIL process is also substrate independent - not requiring heat treatments or complex fabrication procedures - and consequently represents a facile method by which to achieve the complete and smooth coverage of graphene electrodes by HIL, which is critical for successful graphene-based optoelectronic device operation.

As a further demonstration of the broad applicability of the D-HIL structure to graphene electrodes, we also fabricated inverted OPV devices applying graphene as the cathode. With dramatic improvements in the PCE of a single cell in recent OPV developments ${ }^{34-36}$, there have been growing interests in the long term stability/lifetime and the degradation mechanism of organic solar cells (OSCs) for 'real-life' applications. One approach that is frequently proposed is to adopt the inverted solar cell geometry ${ }^{37-39}$ where the nature of charge collection is reversed by using less air-sensitive high work function metals (Ag or $\mathrm{Au}$ ) as the hole collecting back-electrodes. Several works ${ }^{38,40-42}$ reported such inverted architectures using $\mathrm{TiO}_{\mathrm{x}}$ or $\mathrm{ZnO}$ as electron selective layer (ESL) at the ITO front contact and $\mathrm{MoO}_{3}$ or PEDOT:PSS as hole-selective layer (HSL) at the metal back contact due to their efficient charge selectivity.

Up to now, only limited number of works has been demonstrated for graphene as a cathode in OPV: Cox et al. ${ }^{43}$ showed small molecule bi-layer heterojunction (BLHJ) solar cells with laminated single layer graphene but a low PCE (0.02\%). Jo et al. ${ }^{44}$ reported bulk heterojunction $(\mathrm{BHJ})$ devices on work function engineered multi-layer graphene films with an interfacial dipole layer and obtained a PCE of $1.23 \%$, nevertheless, the interfacial polymer layer was not readily available. Here, with the D-HIL, we show efficient graphene cathode-based inverted OSCs using both BLHJ small molecules and BHJ conjugated polymers, and $\mathrm{TiO}_{\mathrm{x}}$ or $\mathrm{ZnO}$ ESL. TiO $\mathrm{x}$ or $\mathrm{ZnO}$ ESL was observed to be critical to the device functionality. In fact recently, a mist-pyrolysis CVD method ${ }^{45}$ was used to deposit $\mathrm{ZnO}$ layers on graphene cathode to achieve inverted OPV cells with $1.55 \%$ PCE. However, we demonstrate a simpler and reproducible method to deposit $\mathrm{TiO}_{\mathrm{x}}$ or $\mathrm{ZnO}$ onto the graphene surface via the D-HIL approach.

The inverted configuration was obtained with simple reversal of normal OPV structures: graphene (or $\mathrm{ITO}) / \mathrm{TiO}_{\mathrm{x}}$ or $\mathrm{ZnO} / \mathrm{C}_{60}$ 

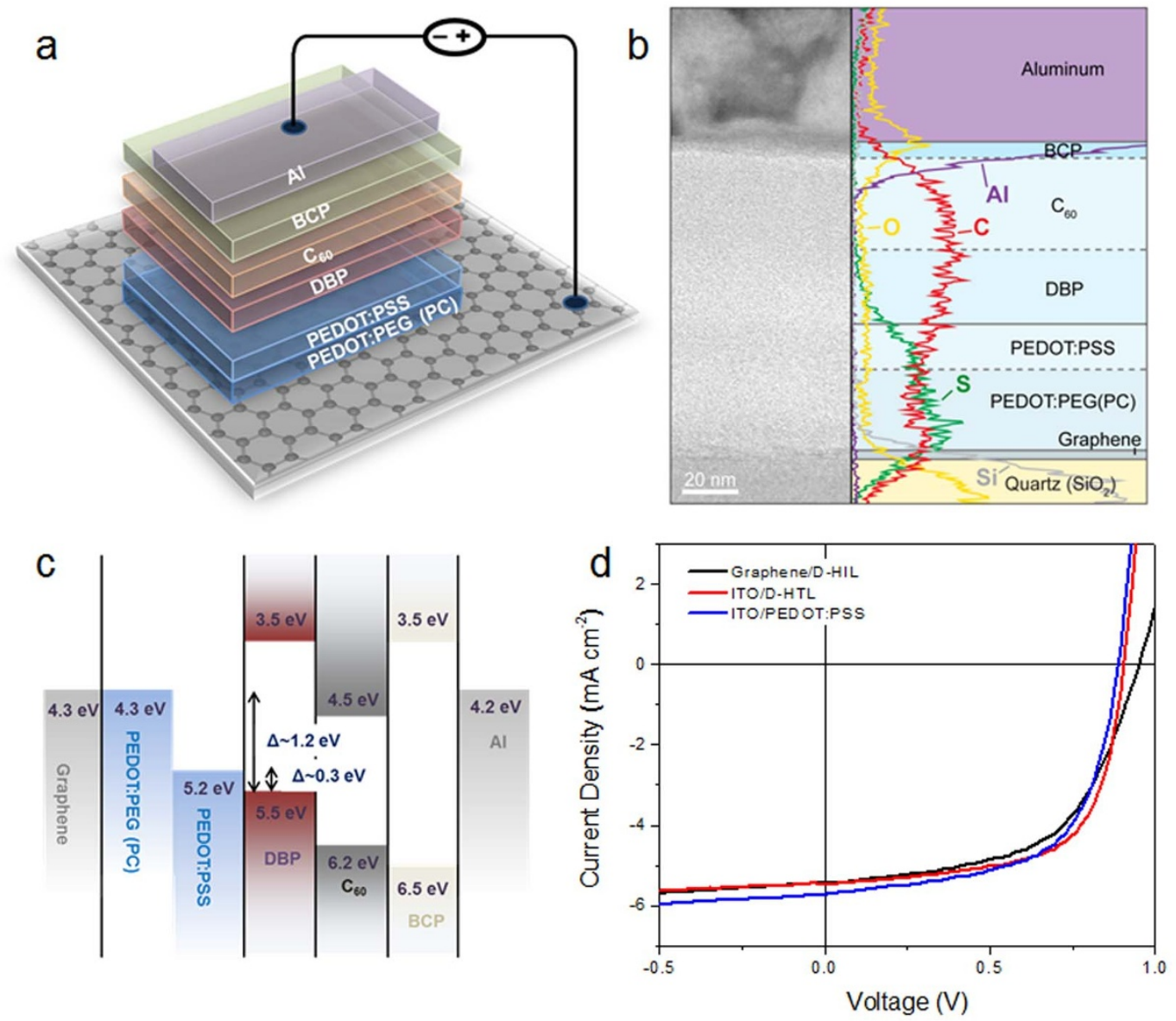

Figure 3 Composition and device performance of typical organic solar cells using graphene anodes. (a) Schematic diagram of the graphene anode OPV architecture: graphene/PEDOT:PEG(PC), $40 \mathrm{~nm} / \mathrm{PEDOT}: P S S, 20 \mathrm{~nm} / \mathrm{DBP}, 25 \mathrm{~nm} / \mathrm{C}_{60}, 40 \mathrm{~nm} / \mathrm{BCP}, 8.5 \mathrm{~nm} / \mathrm{Al}$, $100 \mathrm{~nm}$. (b) Cross-sectional TEM image (left) of the complete device described in (a), with an energy dispersive EDS elemental line scan overlaid on a schematic of the device crosssection (right). Solid lines indicate interfaces clearly identified using TEM and EDS, dashed lines indicate expected locations of interfaces not conclusively resolvable by TEM or EDS, but suggested by differences in contrast. (c) Flat-band energy level diagram of the completed OPV device. (d) Current density vs. voltage $(J-V)$ characteristics of a representative graphene device (using D-HIL, black) compared with ITO reference cells (red and blue) under simulated AM $1.5 \mathrm{G}$ illumination at $100 \mathrm{~mW} \cdot \mathrm{cm}^{-2}$, illustrating comparable performances. $J-V$ characteristics of ITO-based devices with different configurations of HIL, i.e. PEDOT:PSS alone (blue) and the D-HIL (red), are also very similar.

$(40 \mathrm{~nm}) / \mathrm{DBP}(25 \mathrm{~nm}) /$ molybdenum trioxide $\left(\mathrm{MoO}_{3}, 20 \mathrm{~nm}\right) / \mathrm{Ag}$ or $\mathrm{Au}(100 \mathrm{~nm})$. However, rectifying diode characteristics were not observed from these configurations as shown in Fig. 4a unlike devices fabricated on ITO (Fig. 4b). This could be presumably caused by the improper interaction of metal oxides at the graphene interface and the resultant charge recombination due to the lack of chemical reactivity of graphene from the stable $\mathrm{sp}^{2}$ hybridization of carbon atoms ${ }^{46}$. Furthermore, SEM image of $\mathrm{TiO}_{\mathrm{x}}$ on graphene shows non-uniformity in the film compared to that on ITO (Supplementary Fig. S3a, b).

After the graphene surface is modified by the D-HIL, as can be seen in Supplementary Fig. S3d (SEM image of $\mathrm{TiO}_{\mathrm{x}}$ on the D-HIL covered graphene surface), the cracks observed in $\mathrm{TiO}_{\mathrm{x}}$ film spun on bare graphene surface previously are absent after such polymer modification. The surface roughness comes from the roughness of D-HIL, as can be seen by Supplementary Fig. S3c, e. In fact, $\mathrm{TiO}_{\mathrm{x}}$ smoothens the D-HIL layers. Similarly, ZnO film exhibited poor wetting behavior on the pristine graphene surface and improved wetting property was obtained after modification with the D-HIL.

With proper coverage of ESL on the surface-modified graphene cathode, inverted small molecule BLHJ solar cells were fabricated with the following device structure: graphene/D-HIL/TiO ${ }_{\mathrm{x}}(15 \mathrm{~nm}) /$ $\mathrm{C}_{60}(40 \mathrm{~nm}) / \mathrm{DBP}(25 \mathrm{~nm}) / \mathrm{MoO}_{3}(20 \mathrm{~nm}) / \mathrm{Ag}(100 \mathrm{~nm})$. Fabrication and testing procedures are detailed in the Methods section. The resulting $J-V$ characteristics of the inverted devices are shown in Fig. 5a compared with those of devices under normal configurations (graphene/D-HIL/DBP/C $60 / \mathrm{BCP} / \mathrm{Ag}$ ). The results of ITO-based devices (Fig. 5b) are shown as a reference to graphene-based devices: $\mathrm{ITO} / \mathrm{TiO}_{\mathrm{x}} / \mathrm{C}_{60} / \mathrm{DBP} / \mathrm{MoO}_{3} / \mathrm{Ag}$ (inverted) and ITO/PEDOT:PSS/ $\mathrm{DBP} / \mathrm{C}_{60} / \mathrm{BCP} / \mathrm{Ag}$ (normal). As seen from Fig. $5 \mathrm{a}$, insertion of polymeric buffer layers significantly improved the device performance with a desired rectifying diode characteristic, where the performance approaches to the photoresponse of a normal cell: PCE of a representative inverted device was $1.87 \%$ with $\mathrm{J}_{\mathrm{SC}}, \mathrm{V}_{\mathrm{OC}}$, and FF of $3.92 \mathrm{~mA} / \mathrm{cm}^{2}, 0.86 \mathrm{~V}, 0.56$, respectively. The PCE of an inverted geometry was $67 \%$ of that with a normal structure and the $\mathrm{V}_{\mathrm{OC}}$ was comparable to that of normal configuration with slightly less $\mathrm{J}_{\mathrm{SC}}$ and FF. Similar device comparison was observed with ITO-based solar cells: the inverted one has PCE of $2.08 \%$, and is $71 \%$ of the device with normal configuration (PCE: 2.95\%). Analogous device characteristics between inverted and normal structures with ITO electrodes using small molecule as photoactive layers were also reported elsewhere ${ }^{47}$. Further study on the devices with interfacial polymers only, i.e. without $\mathrm{TiO}_{\mathrm{x}}$ ESL (Supplementary Fig. S4), shows a simple resistor-like behavior, confirming that the observed diode characteristic is originated from the effective charge selection and limited charge recombination through the $\mathrm{TiO}_{\mathrm{x}}$ ESL. Using small 

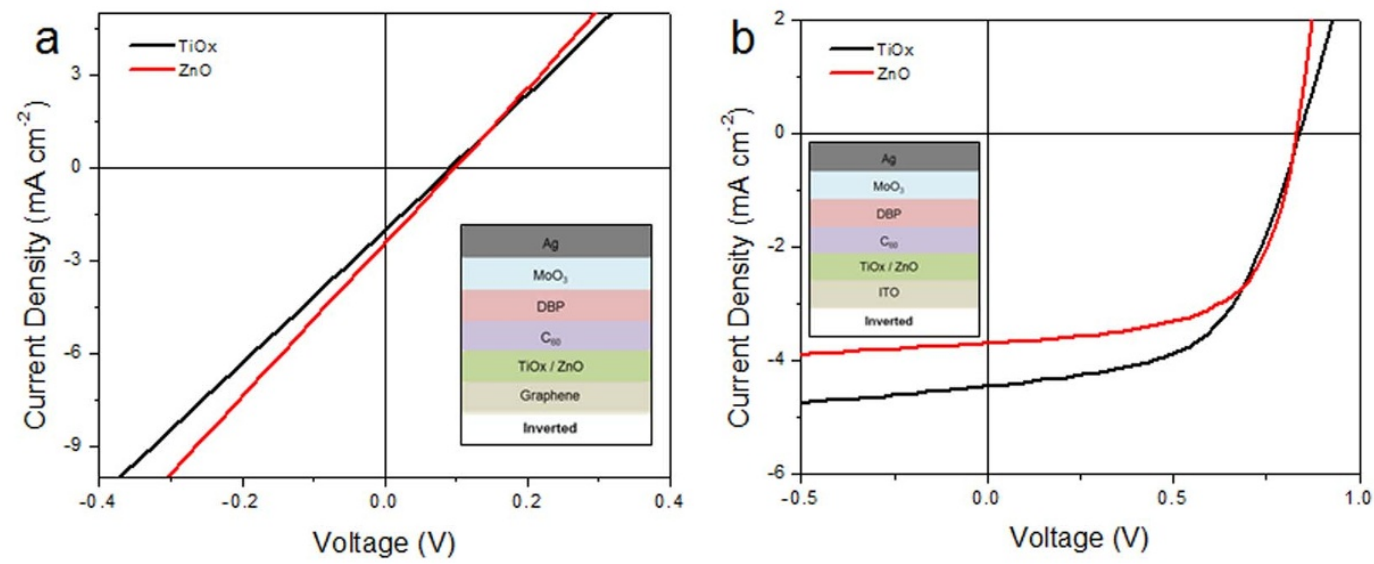

Figure $4 \mid J-V$ measurements of inverted small molecule solar cells with $\mathrm{TiO}_{\mathrm{x}}$ or $\mathrm{ZnO}$ ESL. (a) On pristine graphene electrode and (b) on ITO under AM $1.5 \mathrm{G}$ at $100 \mathrm{~mW} \cdot \mathrm{cm}^{-2}$.

molecules as photoactive materials, the efficiency of the inverted graphene cathode-based solar cell in this work is already higher than devices fabricated with BHJ P3HT:PCBM (poly(3-hexylthiophene): $[6,6]$-phenyl- $\mathrm{C}_{61}$-butyric acid methyl ester) reported elsewhere (PCE: $1.55 \%)^{45}$.

As a further demonstration of the broad applicability of our proposed interface modification approach, we also fabricated graphene cathode-based solar cells using $\mathrm{ZnO}$ film as the ESL. We also note that the synthesis of $\mathrm{ZnO}$ solution is simpler than the preparation of $\mathrm{TiO}_{\mathrm{x}}$ precursor solution (see Methods section) and thus the following works are performed using ZnO ESL. The resulting $J-V$ characteristics are shown in Fig. 6a comparing the device performances of graphene- and ITO-based inverted solar cells using small molecules as photoactive media: the PCEs were $1.20 \%$ and $1.88 \%$, respectively. Our approach was also applicable to BHJ P3HT:PCBM conjugated polymer solar cells which generally result in more efficient devices due to the enhanced interface areas for exciton dissociation. The device structure was graphene/D-HIL/ZnO $(20 \mathrm{~nm}) / \mathrm{P} 3 \mathrm{HT}$ :PCBM $(100 \mathrm{~nm}) / \mathrm{MoO}_{3}(20 \mathrm{~nm}) / \mathrm{Au}(100 \mathrm{~nm})$ with a control device of ITO/ZnO/P3HT:PCBM/ $/ \mathrm{MoO}_{3} / \mathrm{Au}$. The corresponding $J-V$ measurements are shown in Fig. 6b. By using BHJ configuration, we observed improved device performances from both graphene- and ITO-based solar cells with PCEs of $2.27 \%$ and $3.12 \%$, respectively. The photovoltaic performance parameters of the above inverted devices are summarized in Supplementary Table S2.

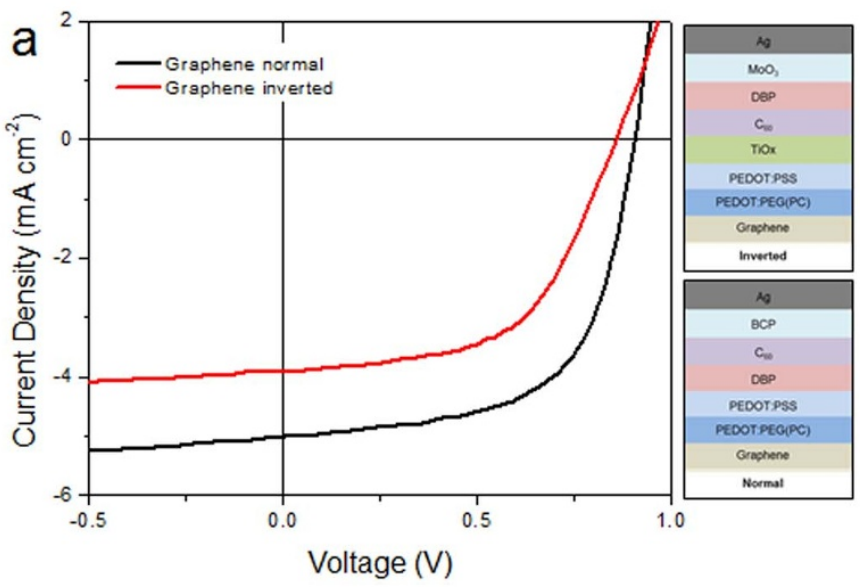

Furthermore, utilizing transparent ITO electrodes by replacing the opaque Ag or Au top electrodes, we successfully fabricated semitransparent OPV devices using graphene cathode as front-window electrodes with ITO anode as back-contact electrodes. Supplementary Fig. S5a shows the result of $J-V$ measurements from semi-transparent solar cells with BHJ P3HT:PCBM photoactive layer: the PCEs were $1.03 \%$ for graphene and $1.58 \%$ for ITO. Although the PCEs of semi-transparent devices were somewhat lower than those of opaque counterparts, most likely due to the optical losses at the ITO side, the successful demonstration of graphene-based inverted semi-transparent OSCs can open up many opportunities such as the buildingintegrated photovoltaics (BIPV) system. Additional results on small molecule based solar cells are shown in Supplementary Fig. S5b. The photovoltaic performance parameters of semi-transparent devices are summarized in Supplementary Table S3. Flat-band energy level diagram of the aforementioned solar cells is all shown in Supplementary Fig. S6.

\section{Discussion}

Our findings show that the reported D-HIL structure serves as a desirable charge transporting layer, enabling the integration of graphene electrodes into OPV devices, which exhibit a number of interesting features. First, we emphasize that our architecture is reproducible from run-to-run, and demonstrates good device yields (252 working devices out of 300 cells) approaching the yield of

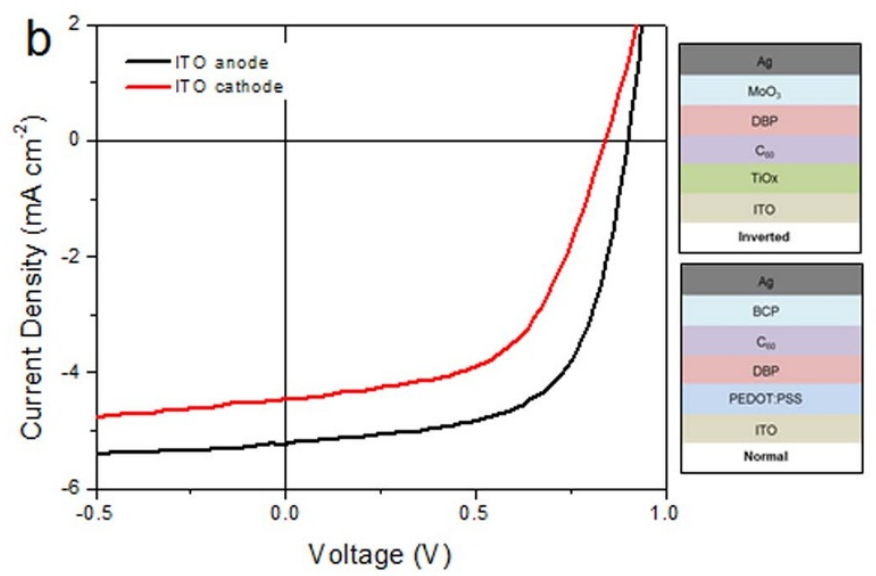

Figure $5 \mid J-V$ characteristics of inverted small molecule solar cells with $\mathrm{TiO}_{\mathbf{x}}$ ESL. (a) On D-HIL modified graphene and (b) on ITO under AM 1.5 G at $100 \mathrm{~mW} \cdot \mathrm{cm}^{-2}$. Device performances with normal geometry are shown for comparison. 

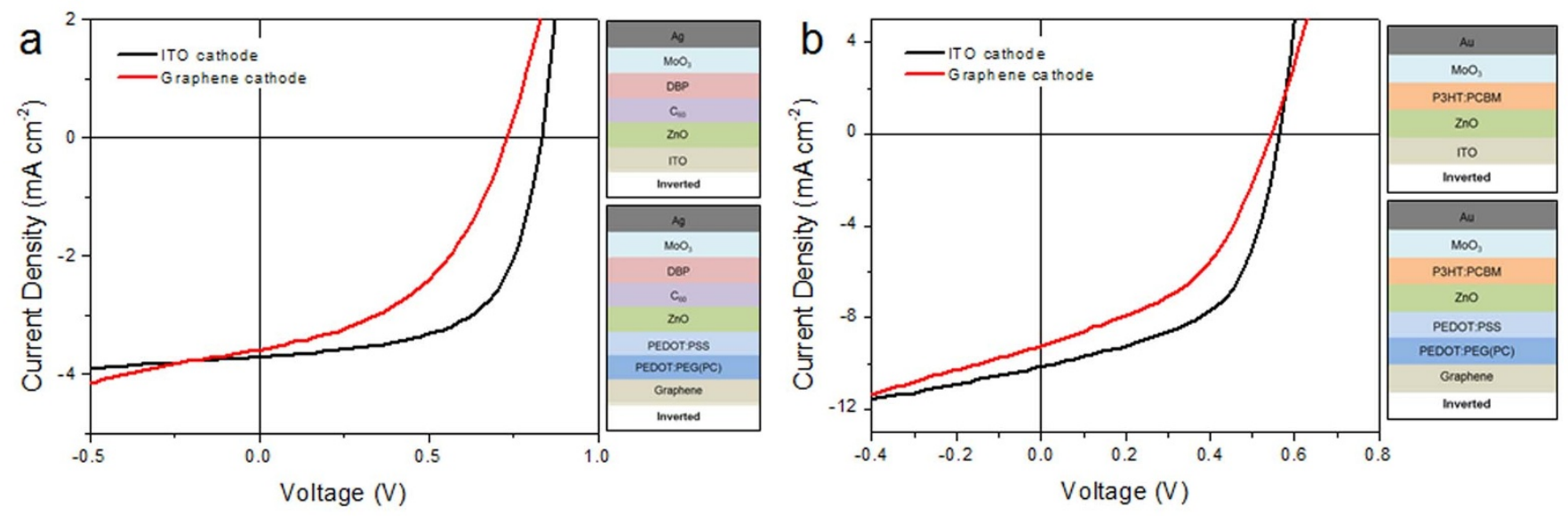

Figure $6 \mid J-V$ characteristics of representative graphene and ITO devices with ZnO ESL. Using (a) small molecule photoactive layers and (b) P3HT:PCBM photoactive layer under AM $1.5 \mathrm{G}$ at $100 \mathrm{~mW} \cdot \mathrm{cm}^{-2}$.

ITO-based reference devices (92 working devices out of 100 cells). Consideration of these two factors, reproducibility and device yield, is essential to the evaluation of solar cells for 'real-world' applications. Indeed, one of the major contributions from the currently reported method is that we can obtain graphene-based device yield close to that with ITO electrodes, and we consider this a big step forward in fabrication of organic solar cells based on graphene electrodes. Moreover, the non-destructive and substrate-independent nature of our approach is conducive to the incorporation of graphene without causing any damage or delamination of the underlying layers. Another important feature regarding the proposed D-HIL configuration is that the performance of OPV solar cells utilizing graphene electrodes is within $10-15 \%$ of ITO reference devices. Notably, this performance is achieved on 'unmodified' and 'undoped' graphene electrodes consisting of only three stacked layers, suggesting the possibility for further improvements. We also note that the lower transparency, conductivity and work function of PEDOT: PEG(PC) than PEDOT:PSS, and the additional junction ${ }^{48}$ introduced in the D-HIL configuration did not limit the device performance at all. In fact, the lower work function of PEDOT:PEG(PC) might be potentially beneficial in applications where the graphene is utilized as an electron injecting cathode layer. Moreover, PEDOT: PEG(PC) is deposited via spin-coating in air similar to PEDOT:PSS, and thus should be able to minimize additional manufacturing costs to the whole device fabrication process. In order to verify the generality of our method, we have also tested organic/inorganic hybrid solar cells ${ }^{49-51}$ and quantum-dot based solar cells ${ }^{52,53}$, all using graphene electrodes and the same interface engineering method introduced in this work, and have observed significant improvements in both device performances and yields, akin to those presented here. The details of these studies will be presented elsewhere ${ }^{54}$.

In summary, we developed a simple method to non-destructively modify the graphene surface using a D-HIL structure, and showed its general applicability to OPV devices with various configurations. This technique ensures smooth and complete coverage of graphene by HIL, which is essential to the successful integration of transparent and conductive graphene electrodes into optoelectronic devices. We demonstrated that graphene-based OPVs utilizing this D-HIL architecture show device performances and yields close to conventional ITO-based devices. From the D-HIL approach, we also demonstrated graphene cathode-based inverted solar cells using $\mathrm{TiO}_{\mathrm{x}}$ or $\mathrm{ZnO}$ as electron transporting and hole blocking layer. The successful demonstration of graphene-based inverted semi-transparent solar cells can be of further interest such as in the BIPV application. This work brings one step forward in the realization of graphene as a viable replacement or alternative to ITO for various optoelectronic device applications.

\section{Methods}

Graphene synthesis and transfer. Graphene films were synthesized via low pressure chemical vapor deposition (LPCVD) using copper foil $(25 \mu \mathrm{m}$ in thickness, ALFA AESAR) as a metal catalyst, as described before in detail ${ }^{17}$. Transfer was performed using poly(methyl methacrylate) (PMMA, 950 A9, Microchem). After etching the Cu foil (CE-100, Transene), graphene films were rinsed with hydrochloric acid and deionized (DI) water. PMMA layer was removed by annealing at $450^{\circ} \mathrm{C}$ for 2 hours under $\mathrm{H}_{2}(600 \mathrm{sccm})$ and $\mathrm{Ar}(400 \mathrm{sccm})$.

OPV device fabrication. Organic active layers (DBP (Luminescence Technology Corp., >99\%), $\mathrm{C}_{60}$ (Sigma Aldrich, 99.9\%), BCP (Luminescence Technology Corp., $>99 \%)$ ), $\mathrm{MoO}_{3}$ (Alfa Aesar, 99.9995\%), and metals contacts (Al (Alfa Aesar, 3.175 mm slug, 99.999\%), Ag (Alfa Aesar, 1-3 mm shot, 99.999\%), Au (Kurt J. Lesker, $3.175 \mathrm{~mm}$ pallets, $99.999 \%)$ ) were thermally evaporated through shadow masks at a chamber pressure of $2 \times 10^{-6}$ Torr at rates of $1.0 \AA / \mathrm{s}$. $\mathrm{C}_{60}$ was purified once before use via thermal gradient sublimation. $\mathrm{DBP}, \mathrm{BCP}, \mathrm{MoO}_{3}, \mathrm{Al}, \mathrm{Ag}$ and $\mathrm{Au}$ were used as received. The ITO (Plasmaterials) is deposited via rf-sputtering in a 3 to 5 mTorr Ar atmosphere. The first $20 \mathrm{~nm}$ of the film was deposited at a rate of $0.01 \mathrm{~nm} / \mathrm{s}$ using a low sputtering power of $7 \mathrm{~W}$ to minimize damage to the underlying $\mathrm{MoO}_{3}$ film, and the remaining $80 \mathrm{~nm}$ of ITO film was deposited at $0.05 \mathrm{~nm} / \mathrm{s}$ and $50 \mathrm{~W}$.

P3HT (Sigma Aldrich) and PCBM (Sigma Aldrich) were dissolved in dichlorobenzene $(30 \mathrm{mg} / \mathrm{ml})$ and mixed at $1: 1 \mathrm{v} / \mathrm{v}$. The mixture was spin-coated at $1000 \mathrm{rpm}$ for $60 \mathrm{~s}$, and annealed at $150^{\circ} \mathrm{C}$ for $45 \mathrm{~min}$ in nitrogen filled glovebox.

PEDOT:PSS (Clevios ${ }^{\mathrm{TM}}$ P VP AI 4083$)$ was filtered $(0.45 \mu \mathrm{m})$, spin-coated at $4000 \mathrm{rpm}$ for $60 \mathrm{~s}$, and annealed at $175^{\circ} \mathrm{C}$ for $5 \mathrm{~min}$ in ambient condition. PEDOT:PEG(PC) in nitromethane (1 wt.\%, Sigma Aldrich) was filtered $(0.2 \mu \mathrm{m})$, spin-coated at $5000 \mathrm{rpm}$ for $60 \mathrm{~s}$ and was spin-dried in ambient condition. The DHIL layer was formed by consecutive spin-coating of PEDOT:PEG(PC) and PEDOT:PSS.

Pre-patterned ITO substrates (Thin Film Devices, $150 \mathrm{~nm}$ thick, $20 \Omega / \mathrm{sq}, 85 \% \mathrm{~T}$ ) were cleaned by sonication in soap water (Micro-90, Cole-Parmer), DI water, acetone and isopropanol, followed by exposure to $\mathrm{O}_{2}$ plasma (100 W, Plasma Preen, Inc.) for $3 \mathrm{~min}$. Patterned graphene substrates were cleaned by annealing at $450^{\circ} \mathrm{C}$ for $30 \mathrm{~min}$ under $\mathrm{H}_{2}(600 \mathrm{sccm})$ and $\mathrm{Ar}(400 \mathrm{sccm})$. The device area defined by the overlap between the top and bottom electrodes was $1.21 \mathrm{~mm}^{2}$.

Sol-gel chemistry for producing $\mathrm{TiO}_{\mathbf{x}}$ layer. A precursor, Titanium(IV) isopropoxide $\left(\mathrm{Ti}\left[\mathrm{OCH}\left(\mathrm{CH}_{3}\right)_{2}\right]_{4}\right)$ was mixed with 2-methoxyethanol

$\left(\mathrm{CH}_{3} \mathrm{OCH}_{2} \mathrm{CH}_{2} \mathrm{OH}\right)$ and ethanolamine $\left(\mathrm{H}_{2} \mathrm{NCH}_{2} \mathrm{CH}_{2} \mathrm{OH}\right)$ in a reflux flask equipped with a condenser and a thermometer. The mixed solution was heated to $80^{\circ} \mathrm{C}$ for $2 \mathrm{hr}$ in a silicon oil bath under magnetic stirring, followed by heating to $120^{\circ} \mathrm{C}$ for $1 \mathrm{hr}$. The two-step heating $\left(80\right.$ and $\left.120^{\circ} \mathrm{C}\right)$ was repeated for a total of $72 \mathrm{hr}^{55}$. The $\mathrm{TiO}_{\mathrm{x}}$ precursor solution was diluted 10 -fold in isopropyl alcohol. The precursor solution was spin-coated in ambient condition at $5000 \mathrm{rpm}$ for $60 \mathrm{~s}$ and heated at $80^{\circ} \mathrm{C}$ for $10 \mathrm{~min}$. The precursor was converted to $\mathrm{TiO}_{\mathrm{x}}$ by hydrolysis and the cooling step was followed for $5 \mathrm{~min}$.

ZnO layer deposition. ZnO layer was prepared by spin-coating $300 \mathrm{mM}$ of Zinc acetate dihydrate and ethanolamine in 2-methoxyethanol solution and annealing at $175^{\circ} \mathrm{C}$ for $10 \mathrm{~min}$. 
Device characterization. Current-voltage characteristics of the OPV devices were recorded in a nitrogen-filled glovebox using a computer-controlled Keithley 6487 picoammeter source-meter. $100 \mathrm{~mW} \cdot \mathrm{cm}^{-2}$ illumination was provided by $150 \mathrm{~W}$ xenon arc-lamp (Newport 96000) filtered by an AM 1.5 G filter. The specular transmittance spectra of polymers were measured on quartz substrates with a Cary 5000 UV-Vis-NIR dual-beam spectrophotometer (Varian). Sheet resistance was measured using a RM3-AR four point probe station from Jandel Engineering LTD.

Structural characterization. The surface morphologies of graphene, PEDOT:PEG(PC), and PEDOT:PSS were characterized using a Digital Instruments Veeco Dimension 3100 atomic force microscope operated in tapping mode. Scanning electron miscroscopy was performed with a Helios Nanolab 600 Dual Beam at $5 \mathrm{kV}$. Preparation of complete device specimens for cross-sectional TEM was performed with a focused-ion-beam (FIB) using a Helios Nanolab 600 Dual Beam FIB Milling System. A JEOL $2010 \mathrm{FEG}$ analytical electron microscope operated at $200 \mathrm{kV}$ was used to obtain bright-field transmission electron microscopy (BF-TEM) images. Energy-dispersive X-ray spectroscopy (EDS) spectra were obtained from the TEM operating in dark-field scanning TEM (DF-STEM) mode.

1. Berger, C. et al. Electronic confinement and coherence in patterned epitaxial graphene. Science 312, 1191-1196 (2006)

2. Bolotin, K. I. et al. Ultrahigh electron mobility in suspended graphene. Solid State Commun. 146, 351-355 (2008).

3. Moser, J., Barreiro, A. \& Bachtold, A. Current-induced cleaning of graphene. Appl. Phys. Lett. 91, 163513 (2007).

4. Bunch, J. S. et al. Electromechanical resonators from graphene sheets. Science 315, 490-493 (2007).

5. Nair, R. R. et al. Fine structure constant defines visual transparency of graphene. Science 320, 1308-1308 (2008).

6. Wang, X. R. et al. N-Doping of Graphene Through Electrothermal Reactions with Ammonia. Science 324, 768-771 (2009).

7. McClure, J. W. Band Structure of Graphite and de Haas-van Alphen Effect. Phys. Rev. 108, 612 (1957).

8. Wallace, P. R. The Band Theory of Graphite. Phys. Rev. 71, 622 (1947).

9. Novoselov, K. S. et al. Two-dimensional atomic crystals. Proc. Natl. Acad. Sci. U. S. A. 102, 10451-10453 (2005).

10. Reina, A. et al. Large Area, Few-Layer Graphene Films on Arbitrary Substrates by Chemical Vapor Deposition. Nano Lett. 9, 30-35 (2009).

11. Li, X. S. et al. Large-Area Synthesis of High-Quality and Uniform Graphene Films on Copper Foils. Science 324, 1312-1314 (2009).

12. Lin, Y. M. et al. 100-GHz Transistors from Wafer-Scale Epitaxial Graphene. Science 327, 662-662 (2010).

13. Park, H., Rowehl, J. A., Kim, K. K., Bulovic, V. \& Kong, J. Doped graphene electrodes for organic solar cells. Nanotechnology 21, 6 (2010).

14. Koppens, F. H. L., Chang, D. E. \& de Abajo, F. J. G. Graphene Plasmonics: A Platform for Strong Light-Matter Interactions. Nano Lett. 11, 3370-3377 (2011)

15. Eda, G., Fanchini, G. \& Chhowalla, M. Large-area ultrathin films of reduced graphene oxide as a transparent and flexible electronic material. Nat. Nanotechnol. 3, 270-274 (2008).

16. Bonaccorso, F., Sun, Z., Hasan, T. \& Ferrari, A. C. Graphene photonics and optoelectronics. Nat. Photonics 4, 611-622 (2010).

17. Park, H., Brown, P. R., Bulovic, V. \& Kong, J. Graphene As Transparent Conducting Electrodes in Organic Photovoltaics: Studies in Graphene Morphology, Hole Transporting Layers, and Counter Electrodes. Nano Lett. 12, 133-140 (2012).

18. De Arco, L. G. et al. Continuous, Highly Flexible, and Transparent Graphene Films by Chemical Vapor Deposition for Organic Photovoltaics. Acs Nano 4 2865-2873 (2010).

19. Wang, Y. W. Y., Tong, S. W., Xu, X. F., Ozyilmaz, B. \& Loh, K. P. Interface Engineering of Layer-by-Layer Stacked Graphene Anodes for High-Performance Organic Solar Cells. Adv. Mater. 23, 1514-1518 (2011).

20. Hwang, J., Amy, F. \& Kahn, A. Spectroscopic study on sputtered PEDOT PSS: Role of surface PSS layer. Org. Electron. 7, 387-396 (2006).

21. Gunes, S., Neugebauer, H. \& Sariciftci, N. S. Conjugated polymer-based organic solar cells. Chem. Rev. 107, 1324-1338 (2007).

22. Peumans, P. \& Forrest, S. R. Very-high-efficiency double-heterostructure copper phthalocyanine/C60 photovoltaic cells. Appl. Phys. Lett. 79, 126-128 (2001).

23. Wang, S. R., Zhang, Y., Abidi, N. \& Cabrales, L. Wettability and Surface Free Energy of Graphene Films. Langmuir 25, 11078-11081 (2009).

24. Choe, M. et al. Efficient bulk-heterojunction photovoltaic cells with transparent multi-layer graphene electrodes. Org. Electron. 11, 1864-1869 (2011).

25. Murakami, T. N. et al. Surface modification of polystyrene and poly(methyl methacrylate) by active oxygen treatment. Colloid Surf. B-Biointerfaces 29, 171-179 (2003).

26. Park, H. et al. Organic Solar Cells with Graphene Electrodes and Vapor Printed Poly(3,4-ethylenedioxythiophene) as the Hole Transporting Layers. ACS Nano 6 , 6370-6377 (2012).

27. Park, H., Shi, Y. M. \& Kong, J. Application of Solvent Modified PEDOT:PSS to Graphene Electrodes in Organic Solar Cells. submitted (2013).
28. Baek, W. H., Choi, M., Yoon, T. S., Lee, H. H. \& Kim, Y. S. Use of fluorine-doped tin oxide instead of indium tin oxide in highly efficient air-fabricated inverted polymer solar cells. Appl. Phys. Lett. 96, 133506 (2010).

29. Lim, F. J., Ananthanarayanan, K., Luther, J. \& Ho, G. W. Influence of a novel fluorosurfactant modified PEDOT:PSS hole transport layer on the performance of inverted organic solar cells. J. Mater. Chem. 22, 25057-25064 (2012).

30. Lipomi, D. J., Tee, B. C. K., Vosgueritchian, M. \& Bao, Z. N. Stretchable Organic Solar Cells. Adv. Mater. 23, 1771-1775 (2011).

31. de Jong, M. P., van Ijzendoorn, L. J. \& de Voigt, M. J. A. Stability of the interface between indium-tin-oxide and poly(3,4-ethylenedioxythiophene)/ poly(styrenesulfonate) in polymer light-emitting diodes. Appl. Phys. Lett. 77, 2255-2257 (2000).

32. Lee, S. T., Gao, Z. Q. \& Hung, L. S. Metal diffusion from electrodes in organic light-emitting diodes. Appl. Phys. Lett. 75, 1404-1406 (1999).

33. Fujishima, D. et al. Organic thin-film solar cell employing a novel electron-donor material. Sol. Energy Mater. Sol. Cells 93, 1029-1032 (2009).

34. Park, S. H. et al. Bulk heterojunction solar cells with internal quantum efficiency approaching 100\%. Nat. Photonics 3, 297-302 (2009).

35 . Chen, H. Y. et al. Polymer solar cells with enhanced open-circuit voltage and efficiency. Nat. Photonics 3, 649-653 (2009).

36. Liang, Y. Y. et al. For the Bright Future-Bulk Heterojunction Polymer Solar Cells with Power Conversion Efficiency of 7.4\%. Adv. Mater. 22, E135-138 (2010).

37. Jorgensen, M., Norrman, K. \& Krebs, F. C. Stability/degradation of polymer solar cells. Sol. Energy Mater. Sol. Cells 92, 686-714 (2008).

38. Hau, S. K. et al. Air-stable inverted flexible polymer solar cells using zinc oxide nanoparticles as an electron selective layer. Appl. Phys. Lett. 92, 253301 (2008).

39. Waldauf, C. et al. Highly efficient inverted organic photovoltaics using solution based titanium oxide as electron selective contact. Appl. Phys. Lett. 89, 233517 (2006).

40. Hayakawa, A., Yoshikawa, O., Fujieda, T., Uehara, K. \& Yoshikawaa, S. High performance polythiophene/fullerene bulk-heterojunction solar cell with a $\mathrm{TiO}_{\mathrm{x}}$ hole blocking layer. Appl. Phys. Lett. 90, 163517 (2007).

41. Tao, C. et al. Performance improvement of inverted polymer solar cells with different top electrodes by introducing a $\mathrm{MoO}_{3}$ buffer layer. Appl. Phys. Lett. 93, 193307 (2008).

42. White, M. S., Olson, D. C., Shaheen, S. E., Kopidakis, N. \& Ginley, D. S. Inverted bulk-heterojunction organic photovoltaic device using a solution-derived $\mathrm{ZnO}$ underlayer. Appl. Phys. Lett. 89, 143517 (2006).

43. Cox, M. et al. Single-layer graphene cathodes for organic photovoltaics. Appl. Phys. Lett. 98, 123303 (2011).

44. Jo, G. et al. Tuning of a graphene-electrode work function to enhance the efficiency of organic bulk heterojunction photovoltaic cells with an inverted structure. Appl. Phys. Lett. 97, 213301 (2010).

45. Shin, K. S. et al. High quality graphene-semiconducting oxide heterostructure for inverted organic photovoltaics. J. Mater. Chem. 22, 13032-13038 (2012).

46. Blake, P. et al. Graphene-based liquid crystal device. Nano Lett. 8, 1704-1708 (2008).

47. Tong, X. R., Lassiter, B. E. \& Forrest, S. R. Inverted organic photovoltaic cells with high open-circuit voltage. Org. Electron. 11, 705-709 (2010)

48. Wang, Z. B. et al. Direct hole injection in to $4,4^{\prime}-\mathrm{N}, \mathrm{N}^{\prime}$-dicarbazole-biphenyl: A simple pathway to achieve efficient organic light emitting diodes. J. Appl. Phys 108, $024510(2010)$

49. Beek, W. J. E., Wienk, M. M. \& Janssen, R. A. J. Hybrid solar cells from regioregular polythiophene and $\mathrm{ZnO}$ nanoparticles. Adv. Funct. Mater. 16 1112-1116 (2006).

50. Greene, L. E., Law, M., Yuhas, B. D. \& Yang, P. D. ZnO-TiO 2 core-shell nanorod/ P3HT solar cells. J. Phys. Chem. C 111, 18451-18456 (2007).

51. Peiro, A. M. et al. Hybrid polymer/metal oxide solar cells based on $\mathrm{ZnO}$ columnar structures. J. Mater. Chem. 16, 2088-2096 (2006).

52. Brown, P. R. et al. Improved Current Extraction from $\mathrm{ZnO} / \mathrm{PbS}$ Quantum Dot Heterojunction Photovoltaics Using a $\mathrm{MoO}_{3}$ Interfacial Layer. Nano Lett. 11 , 2955-2961 (2011).

53. Luther, J. M. et al. Stability Assessment on a 3\% Bilayer PbS/ZnO Quantum Dot Heterojunction Solar Cell. Adv. Mater. 22, 3704-3707 (2010).

54. Park, H. et al. Graphene Cathode-Based ZnO Nanowire Hybrid Solar Cells. Nano Lett. 13, 233-239 (2013).

55. Kim, J. Y. et al. Efficient tandem polymer solar cells fabricated by all-solution processing. Science 317, 222-225 (2007)

\section{Acknowledgements}

The authors gratefully acknowledge the financial support of this work from Eni S.p.A. under the Eni-MIT Alliance Solar Frontiers Center, as well as the use of the materials testing facilities within the MIT Center for Materials Science and Engineering funded by the National Science Foundation under MIT NSF MRSEC Grant No. 581 DMR-08-19762. The authors also gratefully acknowledge Professor Vladimir Bulović for the use of experimental equipment for OPV device fabrication and testing. 


\section{Author contributions}

H.P. and J.K. designed the experiment; H.P. and S.C. carried out the experiment; M.S. did the TEM characterization. S.G. assisted data analysis. H.P. wrote the manuscript and all authors discussed the results and reviewed the manuscript.

\section{Additional information}

Supplementary information accompanies this paper at http://www.nature.com/ scientificreports
Competing financial interests: The authors declare no competing financial interests. License: This work is licensed under a Creative Commons

Attribution-NonCommercial-NoDerivs 3.0 Unported License. To view a copy of this license, visit http://creativecommons.org/licenses/by-nc-nd/3.0/

How to cite this article: Park, H., Chang, S., Smith, M., Gradečak, S. \& Kong, J. Interface engineering of graphene for universal applications as both anode and cathode in organic photovoltaics. Sci. Rep. 3, 1581; DOI:10.1038/srep01581 (2013). 